Draft VERSiON July 8, 2021

Preprint typeset using LATEX style AASTeX6 v. 1.0

\title{
ESTABLISHING $\alpha$ OPH AS A PROTOTYPE ROTATOR: PRECISION ORBIT WITH NEW KECK, CHARA, AND RV OBSERVATIONS
}

Tyler Gardner ${ }^{1}$, John D. Monnier ${ }^{1}$, Francis C. Fekel ${ }^{2}$, Michael Williamson $^{2}$, Fabien Baron $^{3}$, Sasha Hinkley $^{4}$, Michael Ireland ${ }^{5}$, Adam L. Kraus ${ }^{6}$, Stefan Kraus ${ }^{4}$, Rachael M. Roettenbacher ${ }^{7}$, Gail Schaefer ${ }^{3}$, Judit Sturmann $^{3}$, Laszlo Sturmann $^{3}$, Theo Ten BrummelaAR $^{3}$

${ }^{1}$ Astronomy Department, University of Michigan, Ann Arbor, MI 48109, USA

${ }^{2}$ Center of Excellence in Information Systems, Tennessee State University, Nashville, TN 37209, USA

${ }^{3}$ The CHARA Array of Georgia State University, Mount Wilson Observatory, Mount Wilson, CA 91203, USA

${ }^{4}$ University of Exeter, School of Physics and Astronomy, Stocker Road, Exeter, EX4 4QL, UK

${ }^{5}$ Research School of Astronomy \& Astrophysics, Australian National University, Canberra ACT 2611, Australia

${ }^{6}$ Department of Astronomy, University of Texas at Austin, Austin, TX, 78712, USA

${ }^{7}$ Yale Center for Astronomy and Astrophysics, Department of Physics, Yale University, New Haven, CT 06520, USA

\begin{abstract}
Alpha Ophiuchi (Rasalhague) is a nearby rapidly rotating A5IV star which has been imaged by infrared interferometry. $\alpha$ Oph is also part of a known binary system, with a companion semi-major axis of $\sim 430$ milli-arcseconds and high eccentricity of 0.92 . The binary companion provides the unique opportunity to measure the dynamical mass to compare with the results of rapid rotator evolution models. The lack of data near periastron passage limited the precision of mass measurements in previous work. We add new interferometric data from the MIRC combiner at the CHARA Array as well as new Keck adaptive optics imaging data with NIRC2, including epochs taken near periastron passage. We also obtained new radial velocities of both components at Fairborn Observatory. Our updated combined orbit for the system drastically reduces the errors of the orbital elements, and allows for precise measurement of the primary star mass at the few percent level. Our resulting primary star mass of $2.20 \pm 0.06 \mathrm{M}_{\odot}$ agrees well with predictions from imaging results, and matches evolution models with rotation when plotting on an HR diagram. However, to truly distinguish between nonrotating and rotating evolution models for this system we need $\sim 1 \%$ errors on mass, which might be achieved once the distance is known to higher precision in future Gaia releases. We find that the secondary mass of $0.824 \pm 0.023 \mathrm{M}_{\odot}$ is slightly under-luminous when compared to stellar evolution models. We show that $\alpha \mathrm{Oph}$ is a useful reference source for programs that need \pm 1 milli-arcsecond astrometry.
\end{abstract}

Keywords: binaries: close, technique: interferometry

\section{INTRODUCTION}

Observational methods that provide fundamental properties of stars are crucial for benchmarking stellar evolution models. Binary stars are frequently targeted since they provide a direct measurement of stellar mass to compare with models. Visual binary orbits alone provide the sum of masses in the system through Kepler's laws, and combining visual orbits with velocity information from double-lined spectroscopic orbits gives orbital parallax and masses of individual components of the system. Imaging of stellar surfaces is another important measurement which can reveal oblateness, latitude dependencies on radius / temperature, and spots and other surface features (for a review on imaging of stars see e.g. van Belle 2012). Due to large distances, however, most stars are unresolved point sources to traditional single aperture telescopes. Long-baseline optical interferometers with $<1$ milli-arcsecond (mas) resolution are needed to image the largest stars in the sky. Rapidly rotating stars are particularly interesting targets for imaging with optical interferometers, since imaging can provide measurements of inclination, gravity darkening, differential rotation, as well as stellar mass and age estimates (e.g. Monnier et al. 2007, 2012; Zhao et al. 2009; Domiciano de Souza et al. 2014).

$\alpha$ Oph (Rasalhague, HD 159561) is a nearby, bright, A5IV star which is both a rapid rotator (rotating at $~ 90 \%$ of its breakup velocity) and in a $<1$ arcsecond visual binary system. This combination provides a unique opportunity 
to benchmark mass estimates from imaging models of rapid rotators to the direct measurement provided from the binary orbit. McCarthy (1983) was the first to resolve the $\sim 8.6$-year secondary component of $\alpha$ Oph with speckle interferometry, and the orbit has been monitored since then. Hinkley et al. (2011) carried out a thorough investigation of the visual orbit available at the time from adaptive optics (AO) imaging, combined with photometry and photocenter motion in order to obtain a measurement of both components' masses at $\sim 10 \%$ uncertainty level. Their masses for the $\mathrm{A}$ and $\mathrm{B}$ components of $2.40_{-0.37}^{+0.23} \mathrm{M}_{\odot}$ and $0.85_{-0.04}^{+0.06} \mathrm{M}_{\odot}$ were lower than previous measurements (Gatewood 2005). These lower mass values were in better agreement with the results provided by rapid rotator models from interferometric imaging with the Michigan Infra-Red Combiner (MIRC) at the Georgia State University Center for High Angular Resolution Astronomy (CHARA) Array, which estimated a mass of $2.1 \pm 0.02 \mathrm{M}_{\odot}$ for the primary using stellar evolution models, or 1.7-2.2 $\mathrm{M}_{\odot}$ using their new oblateness model method (Zhao et al. 2009). Bailey et al. (2020) utilized the polarization signal arising from rapid rotation in order to estimate a mass of $2.0 \pm 0.4 \mathrm{M}_{\odot}$ for the primary, also consistent with the lower mass of Hinkley et al. (2011). $\alpha$ Oph has also been targeted for asteroseismic modeling efforts (Monnier et al. 2010), in order to learn more about the interior of stars rotating near breakup velocity. Since mass is critical to understanding a star's evolution and physical properties, it is imperative that we precisely measure the mass of well-studied systems when possible.

Though the binary orbit semi-major axis is $\sim 400$ milli-arcseconds (mas), the separation of the components is $<20$ mas near periastron since eccentricity is very high at $e=0.92$. Up until this work there was no orbital data near periastron passage, which is crucial to measure mass at the few percent precision level needed to check rotator models at high precision. The updated orbit of Hinkley et al. (2011) predicted the time of periastron passage, and we obtained new interferometric and AO imaging data near the passage to improve mass precision. We also obtained double-lined radial velocity (RV) data which allows us to directly measure orbital parallax of the system as well as individual masses. Hence our mass measurements are purely dynamical and do not rely on any outside measurement or modelbased result. Our new orbit for $\alpha$ Oph serves as a benchmark test of rapidly rotating stellar models. $\alpha$ Oph is bright $(V=2.1)$ and easily observable to most telescopes which can observe bright stars. Our new well-covered orbit allows for precise astrometry prediction across all position angles of the orbit, making this target a good standard astrometric source.

In Section 2 we describe our new observations from interferometry, AO imaging, and spectroscopy. Section 3 details our combined visual + RV orbit fitting model for the binary system. In Section 4 we present our new visual and spectroscopic orbit along with physical parameters and masses for each component. We plot these stars on an HR diagram in Section 5. Finally, we comment on the orbital precision and use of $\alpha$ Oph as an astrometric reference in Section 6 .

\section{OBSERVATIONS AND DATA REDUCTION}

\subsection{MIRC at the CHARA Array}

We obtained two previously unpublished epochs of $\alpha$ Oph near periastron in 2012 using the high angular resolution of MIRC. We attempted observations again during the periastron passage of 2020, though poor weather during our observing run prevented us from obtaining any data. MIRC is a H-band combiner of six 1-meter telescopes at the CHARA Array. The CHARA Array is an optical/near-IR interferometer with baselines up to 330 meters (ten Brummelaar et al. 2005). The MIRC instrument is described in detail in Monnier et al. (2006) and Che et al. (2010, 2012). The MIRC combiner measures visibilities and closure phase of our targets. Calibrator stars are observed between science observations to measure visibility loss due to time-variable factors such as atmospheric coherence time, differential dispersion, and birefringence in the beam train. The MIRC datasets were reduced with the standard MIRC data pipeline in IDL described in previous MIRC papers (e.g. Monnier et al. 2012). The star $\gamma$ Oph $\left(\Theta_{\mathrm{UD}}=\right.$ $0.571 \pm 0.040$ milliarcseconds; Source: Jean-Marie Mariotti Center Searchcal tool, Chelli et al. 2016) was used to calibrate the instrumental transfer function for all MIRC $\alpha$ Oph observations presented here.

For each MIRC night we fit to the following binary star model of complex visibility, $V$, in order to measure binary position (Herbison-Evans et al. 1971; Boden et al. 1999, e.g.):

$$
V=\frac{V_{1}+\Gamma f V_{2} e^{-2 \pi i(u \alpha+v \delta)}}{1+f} .
$$

For determining simply the binary parameters, a detailed image of the nearly "edge-on" rapidly-rotating rotating primary star (see Zhao et al. 2009) is not required. Here, $\alpha$ Oph A was approximated as a uniformly-bright elliptical disk (position angle of -53.88 degrees from Zhao et al. 2009) and is represented by $V_{1}$ in Equation 1 . $\alpha$ Oph $\mathrm{B}$ was treated as an unresolved star represented by $V_{2}$. Other free parameters in fit were the binary separation in right 
ascension (R.A., $\alpha$ ) and declination (DEC, $\delta$ ); a monochromatic flux ratio between the two components $f$; as well as a bandwidth smearing correction $\Gamma=\operatorname{sinc}[b(u \alpha+v \delta)]$, where $b=1 / R$ and $R$ is the spectral resolution of the spectrograph. As is standard, the location of each datum on the uv-plane is denoted by parameters $u$ and $v$. We fit to the calibrated squared-visibility and the closure phase. We investigated whether it was acceptable to use this simple symmetric intensity model for the primary rather than the full gravity-darkenened oblate spheroid model when measuring the binary separation. We calculated the expected photocenter shift due to the slight asymmetry in the intensity distribution from the gravity darkening effect using the full model of Zhao et al. (2009), and found the model shift is only 4 micro-arcseconds away from the true center of mass, a small systematic error that we can safely ignore here. Errors on astrometry are estimated by deriving a $\chi^{2}$ surface for a grid in relative R.A. and DEC and finding the 1- $\sigma$ confidence contour. To remain consistent with the AO imaging data, we convert this confidence contour to an error in position angle and separation. Results from the two MIRC epochs, along with the rest of the astrometry data, are presented in Table 1.

\subsection{Keck Adaptive Optics Imaging}

The $\alpha$ Oph system was observed with the Keck-II adaptive optics system and the facility AO imager NIRC2 in six previously-unpublished epochs obtained between 2002 March 27 and 2014 June 10. All observations were taken using the narrow-band camera with several different choices of filters, typically using a subarray to shorten exposure times and minimize saturation. We processed these images following the general procedures described in Kraus et al. (2016), performing a linearity correction, subtracting mode-matched dark frames, and dividing by the most contemporaneous flatfield available for the filter used in each observation. For the imaging observations, we also performed "destriping" to remove spatially correlated readnoise that is mirrored in the 4 quadrants of the NIRC2 detector, as well as identifying cosmic rays and interpolating over them. Finally, we flagged all saturated pixels so they would not be used in our PSF-fitting analysis.

For four of the six epochs, the $\alpha$ Oph components are well-resolved in images and could be fit with a $\chi^{2}$ minimization of a double-source model. Following Kraus et al. (2016), for each frame we iteratively fit the separation, PA, and contrast of the binary pair, and then tested the 1000 most contemporaneous single-star images from the archive to identify the optimal empirical template. We repeated these steps until the same PSF template yielded the lowest $\chi^{2}$ for two consecutive iterations. Using the pixel positions corresponding to these astrometric measurements, the geometric distortion of NIRC2 was corrected using the algorithm described in Yelda et al. (2010) and then the relative astrometry was reported in Table 1. For each epoch, we report the mean separation and PA for all frames in the given filter. The uncertainty represents the RMS of the observed frames, added in quadrature with the systematic uncertainty in separation $\left(\sigma_{\rho}=1.4\right.$ mas $)$ and in PA $\left(\sigma_{P A}=\arctan \left(\frac{1.4 m a s}{\rho}\right)\right)$ that results from the residual uncertainty in the distortion solution of Yelda et al. (2010). The systematic uncertainties in the pixel scale and detector orientation are negligible compared to this term.

Two of the archival epochs (both in 2012 April) occurred very close to periastron passage with separation less than the diffraction limit of the telescope. In order to recover high-precision separations in this situation, the 18-hole aperture mask within NIRC2 was used following procedures discussed in other recent papers (e.g., Ireland et al. 2008; Kraus \& Ireland 2012; Willson et al. 2016; Rizzuto et al. 2020). By fitting a precise binary model to the interferometric visibilities and closure phases formed by the aperture mask, a precise component separation can be extracted after fixing the component flux ratio established from previous wide-separation measurements. For 2012 Apr 5 we have a measurement taken both at Keck and at the CHARA Array which show good agreement within errors of the binary position taken with two different instruments.

\subsection{Fairborn Observatory Radial Velocities}

Between 2011 and 2020 we obtained 145 new radial velocity (RV) data points for the primary component of $\alpha$ Oph, and $107 \mathrm{RVs}$ for the secondary. This period covers two periastron passages of $\alpha$ Oph, crucial for obtaining masses at the few percent level. These data were taken with the Tennessee State University $2 \mathrm{~m}$ Automated Spectroscopic Telescope (AST) and its echelle spectrograph at the Fairborn Observatory in southeast Arizona (Eaton \& Williamson 2007). The detector was a Fairchild $486 \mathrm{CCD}$ that has a $4 \mathrm{~K} \times 4 \mathrm{~K}$ array of $15 \mu \mathrm{m}$ pixels (Fekel et al. 2013). The spectra have a resolution of $0.24 \AA$, corresponding to a resolving power of 25000 at $6000 \AA$, and cover a wavelength range from 3800 to $8260 \AA$. After acquiring a couple test spectra of $\alpha$ Oph and trying to measure RVs, we settled on the following observing sequence to optimize RV measurement of the very broad lined primary star and the extremely weak lines of its very faint companion. Because of the brightness of the $\alpha$ Oph system, we typically acquired 40 consecutive $30 \mathrm{sec}$ observations, which we then summed together into a single spectrum that has a significantly improved signal-to-noise 
ratio (SNR).

For the A5 IV primary we initially tried to measure the velocities of the lines compiled in our A star line list. This list primarily consists of singly ionized elements of Fe and Ti. Unfortunately, we were unable to obtain usable RVs with the lines in this list due to a combination of factors. The most problematic one is the very high rotational velocity of the A star, $228 \mathrm{~km} \mathrm{~s}^{-1}$ (Royer et al. 2002), which causes the lines to be extremely broad and shallow. This makes it much more difficult to measure the velocity centers of the profiles compared with the measurement of a narrow-lined star. Another significant problem is that the very large rotational broadening of the lines greatly increases the likelihood of blending with nearby lines. These problems are illustrated in Figure 1. Shown is an echelle order centered at about $5135 \AA$. Tick marks indicate the rest positions of individual lines in our A star line list. While this order includes the relatively strong $\mathrm{Mg}$ I lines, they are unusable because of blending problems and the other lines are extremely weak. Additional problems with RV measurement, exacerbated by the large rotational broadening, occur because of difficulties with continuum rectification and the fact that the ends of the echelle orders have lower SNRs.

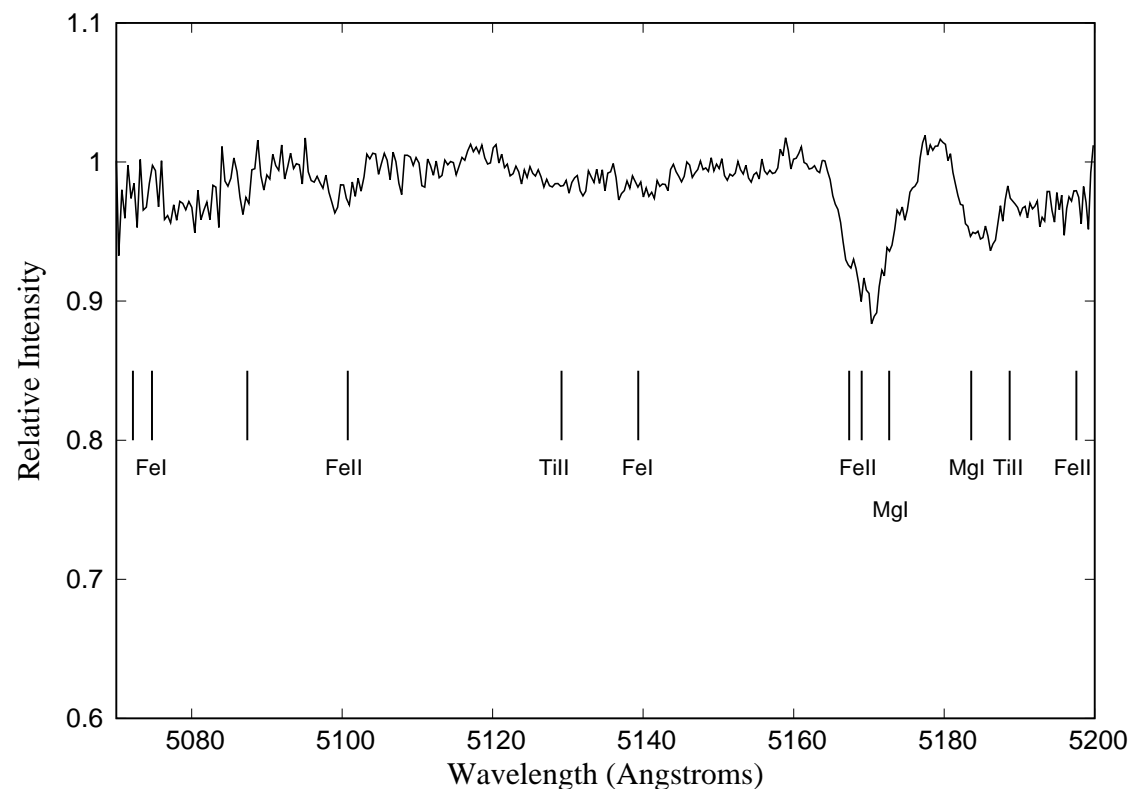

Figure 1. We show an echelle order centered at about $5135 \AA$, for an $\alpha$ Oph spectrum taken with the $2 \mathrm{~m}$ AST at Fairborn Observatory. Due to the high rotational velocity of the A star and blending issues with nearby lines, we were unable to use our A-star lines list to obtain RV. Tick marks indicate the rest positions of individual lines in the list. Though the Mg I lines appear relatively strong in this order, they are unusable due to blending problems with nearby lines. The other lines are too weak to measure reliably. As discussed in the text, we instead determined RVs from the $\mathrm{H} \beta$ line of the primary.

Thus, instead of velocities determined from lines in the A star list, we individually measured the hydrogen lines, $\mathrm{H} \alpha, \mathrm{H} \beta$, and $\mathrm{H} \gamma$. In addition to producing higher SNR, summing the consecutive spectra smoothed out the rapidly varying ripples in the hydrogen profiles that are presumably caused by pulsation. Of the three lines, $\mathrm{H} \gamma$ is well into the blue part of the spectrum where the throughput of our echelle system and detector is low, and there are an increasing number of metal lines that cause blending problems. $\mathrm{H} \alpha$ is near the end of two echelle orders, where there is a lower $\mathrm{SNR}$, and there are increased problems with continuum rectification. The $\mathrm{H} \beta$ line is closer to the middle of its echelle order than $\mathrm{H} \alpha$ is, and RVs of $\mathrm{H} \beta$ result in the orbit that is most consistent with the secondary velocities. Thus, for the primary we choose to adopt the RVs determined from the $\mathrm{H} \beta$ line. However, we note that continuum rectification problems can also affect this broad line and may be at least partly responsible for the significant center-of-mass velocity difference that we find between its orbit and that of the secondary.

Fekel et al. (2009) provided a general explanation of our usual velocity measurement procedure. In particular, for the secondary of $\alpha$ Oph we used our solar-type star line list with which we were able to detect the extremely weak secondary component. That component has a mean line depth of just 0.002 and so is barely detectable in most of our summed spectra. The solar list contains 168 lines in the wavelength range 4920-7100 $\AA$. Given the weakness of average line profile, we chose to report RVs as whole numbers. RVs for the primary and secondary components are reported in Table 2. 


\section{ORBIT FITTING}

Once we have our measured binary separations and position angles for each night, we are able to fit a Keplerian orbit to the data. The Campbell elements $(\omega, \Omega, e, i, a, T, P)$ describe the Keplerian motion of one star of a binary system relative to the other. Those symbols have their usual meanings (Wright \& Howard 2009, e.g.) where $\omega$ is the longitude of the periastron, $\Omega$ is the position angle of the ascending node, $e$ is the eccentricity, $i$ is the orbital inclination, $a$ is angular semi-major axis, $T$ is a time of periastron passage, and $P$ is orbital period. When including RV data, we also fit to the two semi-amplitudes $K_{A}$ and $K_{B}$ and system velocity $\gamma$. Note that as described in Section 2, there are two rather different system velocities for the two components due at least in part to continuum normalization because of the use of the very broad hydrogen spectral line to compute the RVs of the primary. The longitude of periastron $\omega$ is traditionally reported for the secondary when fitting to visual binary orbits alone. The convention when combining RV orbits is to report $\omega$ of the primary, which is flipped by $180^{\circ}$. For visual orbits, there is a 180 degree ambiguity between $\omega$ and $\Omega$. Our RV information breaks this degeneracy.

For nonlinear least-squares fitting, we use the Thiele-Innes elements to describe our Keplerian orbits. As described in Wright \& Howard (2009), these elements convert $(\omega, \Omega, i, a)$ to linear parameters (A, B, F, G). We use the Python lmfit package for non-linear least-squares fitting of our data (Newville et al. 2016). The Python astropy (Astropy Collaboration et al. 2013, 2018) package is also extensively used in our fitting routines. Error bars for the fitted orbital parameters are normally estimated in lmfit from the covariance matrix, but since the orbital elements $P$ and $e$ are nonlinear we instead determine posterior distributions on our orbital parameters with a Markov chain Monte Carlo (MCMC) fitting routine. We carry out MCMC fitting using the Python package emcee developed by Foreman-Mackey et al. (2013). We use our best-fit orbital elements as a starting point for our $2^{*} \mathrm{~N}_{\text {params }}$ walkers, where the starting point for each walker is perturbed about its best fit value. We assume uniform priors on all of our orbital elements within physically allowed parameter space (i.e. eccentricity is restricted between $0-1, \omega$ and $\Omega$ between $0-360^{\circ}$, inclination between $0-180^{\circ}$, no negative values allowed for semi-major axis, period, periastron passage, semi-amplitudes). The quoted error bars on our orbital elements in Table 3 are the standard deviations of the posterior distributions, and corner plots of the posteriors for the inner and outer orbits show correlations between parameters.

\section{NEW VISUAL AND SPECTROSCOPIC ORBIT}

Table 1 compiles all of our previously unpublished data that we use to fit our visual orbit of $\alpha$ Oph. This includes our 2 CHARA/MIRC interferometry and 6 Keck/NIRC2 AO epochs. Table 2 presents our new double-line RV data from Fairborn Observatory. In Table 3 we give the best-fit orbital elements when fitting to astrometry alone, RV alone, and the combined fit. We show our best-fit visual orbit in Figure 2 which also plots the data points from Hinkley et al. (2011), and our best fit RV orbit is shown in Figure 3. For our reported best-fit orbital elements we only fit to our updated high precision data, as this data was observed and analyzed in a systematic and consistent way. Though we checked that most of the historical data points from Hinkley et al. (2011) are consistent with our orbit (i.e. within the quoted 1-sigma error values). The exception are the data points in that paper which were taken with Project 1640 and PHARO instruments at Palomar Observatory, which are multiple sigma off from our best-fit orbit. This is not surprising for Project 1640, as the astrometry returned by the instrument was not yet well understood at the time of observation (private communication with authors). The PHARO data points had error values that were much smaller than the rest of the data from that paper, and there is some hint that these errors may be underestimated. Pope et al. (2016) measured a new epoch on $\alpha$ Oph from PHARO, and Table 1 of that paper shows that the astrometry solution varies by $>10$ mas depending on the fitting method used. This is significantly larger that the $\sim 1-3$ mas error bars given in Hinkley et al. (2011) for the PHARO data. Figure 2 designates the PHARO data points separately from the rest of the Hinkley et al. (2011) data points, to highlight the fact that they do not fit well like the rest of this data. Though we do not include any of this older data in our fit, we checked that including them in the fit does not significantly change our orbital elements or masses within error bars.

With a combined RV and visual orbit, we are able to compute the distance and masses of both components of the system. We present these values in Table 4. Our best-fit distance is $14.80 \pm 0.13 \mathrm{pc}$, which is consistent within error bars with the Hipparcos parallax of $14.90 \pm 0.24$ pc (van Leeuwen 2007). Our best-fit mass values for the primary and secondary are $2.20 \pm 0.06 \mathrm{M}_{\odot}$ and $0.824 \pm 0.023 \mathrm{M}_{\odot}$ respectively. These mass errors are both at the $2.7 \%$ level, which is a significant improvement on the $\sim 10 \%$ mass errors presented in Hinkley et al. (2011), though our values are consistent with that work. This precision allows for a more thorough check to rapid rotator evolution models. Our mass on the primary is just barely outside the 1-sigma error bar of the $2.1 \pm 0.02 \mathrm{M}_{\odot}$ prediction from evolution models using the MIRC imaging results of Zhao et al. (2009). It is consistent with the prediction in that work of 1.7-2.2 $\mathrm{M}_{\odot}$ using their 
oblateness method to estimate mass. Our mass value for the primary also agrees well with polarization work from Bailey et al. (2020), though the precision is lower for that work at $2.0 \pm 0.4 \mathrm{M}_{\odot}$. Our mass values for the primary are significantly lower than previous literature values of $2.84 \pm 0.19 \mathrm{M}_{\odot}$ (Gatewood 2005) and $4.9 \mathrm{M}_{\odot}$ (Kamper et al. 1989), which did not agree well with the rapid rotator model results. The addition of data near periastron passage is likely the cause of the discrepancy with previous literature orbit results, and is indeed crucial for constraining eccentric binary orbits.

Our best-fit orbital elements of $a, i, e, \omega$, and $\Omega$ agree well within error bars with Hinkley et al. (2011), though we note that due to better coverage our values are much more tightly constrained. Hinkley et al. (2011) chose to fix their orbital period to 3148.4 days from Gatewood (2005), which is $\sim 9$ days larger than our best-fit value of $3139.72 \pm 0.28$ when combining the new spectroscopic orbit with the visual orbit. Reported orbital period in literature range from 3109-3165 days (Kamper et al. 1989; Augensen \& Heintz 1992; Gatewood 2005), so our value is consistent with this spread though much more tightly constrained. Corner plots from the posteriors of our MCMC fitting routine are shown in Figure A1. Our errors are derived from the 1- $\sigma$ standard deviation in these posterior distributions, and the plots show correlation between parameters. Since this is an eccentric orbit, there are correlations between $e$ with parameters $\omega, \Omega$, and $T$ which can be seen in our plots.

Similar to Hinkley et al. (2011), we are able to compute the mutual inclination between the primary star's rotation axis and the plane of the binary orbit from the equation of Fekel (1981). Using the values in Zhao et al. (2009) of the imaged rotator model and our updated binary orbit, we find a mutual inclination of either $42.7 \pm 0.6^{\circ}$ or $133.0 \pm 0.6^{\circ}$. Though our orbit is fully characterized with RV data included, the degeneracy in mutual inclination comes from the unknown spin polarity of the primary. In either case, the mutual inclination is significantly non co-planar. Combined with the high eccentricity of the binary orbit, this perhaps suggests early interaction in the star formation phases between the binary orbit and the primary rotation angular momentum.

Table 1. $\alpha$ Oph Astrometry

\begin{tabular}{lcccc}
\hline \multicolumn{1}{c}{ UT Date } & JD & sep (mas) & P.A. $\left({ }^{\circ}\right)$ & Instrument \\
\hline 2002 Mar 27 & 2452360.95 & $545.2 \pm 1.9$ & $233.61 \pm 0.17$ & KECK-II/NIRC2 \\
2010 Apr 26 & 2455313.15 & $635.0 \pm 1.5$ & $236.70 \pm 0.13$ & KECK-II/NIRC2 \\
2012 Apr 5 & 2456022.952 & $22.54 \pm 0.12$ & $104.31 \pm 0.08$ & CHARA/MIRC \\
2012 Apr 5 & 2456023.05 & $20.7 \pm 2.9$ & $100.8 \pm 2.3$ & KECK-II/NIRC2 \\
2012 Apr 14 & 2456032.15 & $25.5 \pm 1.0$ & $36.5 \pm 1.7$ & KECK-II/NIRC2 \\
2012 May 10 & 2456057.939 & $56.19 \pm 0.05$ & $302.95 \pm 0.03$ & CHARA/MIRC \\
2013 Aug 7 & 2456511.86 & $495.6 \pm 1.5$ & $253.87 \pm 0.16$ & KECK-II/NIRC2 \\
2014 Jun 10 & 2456819.06 & $641.6 \pm 1.5$ & $249.81 \pm 0.13$ & KECK-II/NIRC2 \\
\hline
\end{tabular}

Table 2. $\alpha$ Oph Radial Velocities ${ }^{\text {a }}$

\begin{tabular}{lcc}
\hline $\mathrm{HJD}^{\mathrm{b}}$ & $R V_{A}\left(\mathrm{~km} \mathrm{~s}^{-1}\right)^{\mathrm{c}}$ & $R V_{B}\left(\mathrm{~km} \mathrm{~s}^{-1}\right)^{\mathrm{d}}$ \\
\hline 2455847.6533 & 7.8 & - \\
2455927.0572 & 10.2 & - \\
2455930.0570 & 10.0 & - \\
2455935.0558 & 11.5 & - \\
2455947.0524 & 12.1 & - \\
2455957.0473 & 11.9 & - \\
2455967.0446 & 10.9 & - \\
2455976.0409 & 10.8 & - \\
2455991.0334 & 12.4 & - \\
$\ldots$ & $\ldots$ & $\ldots$ \\
\hline
\end{tabular}

$a_{\text {Full table in Appendix A }}$

${ }^{b}$ HJD $=$ Heliocentric Julian Date 
${ }^{c}$ Errors on primary are $1.4 \mathrm{~km} \mathrm{~s}^{-1}$.

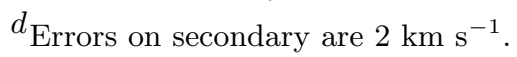
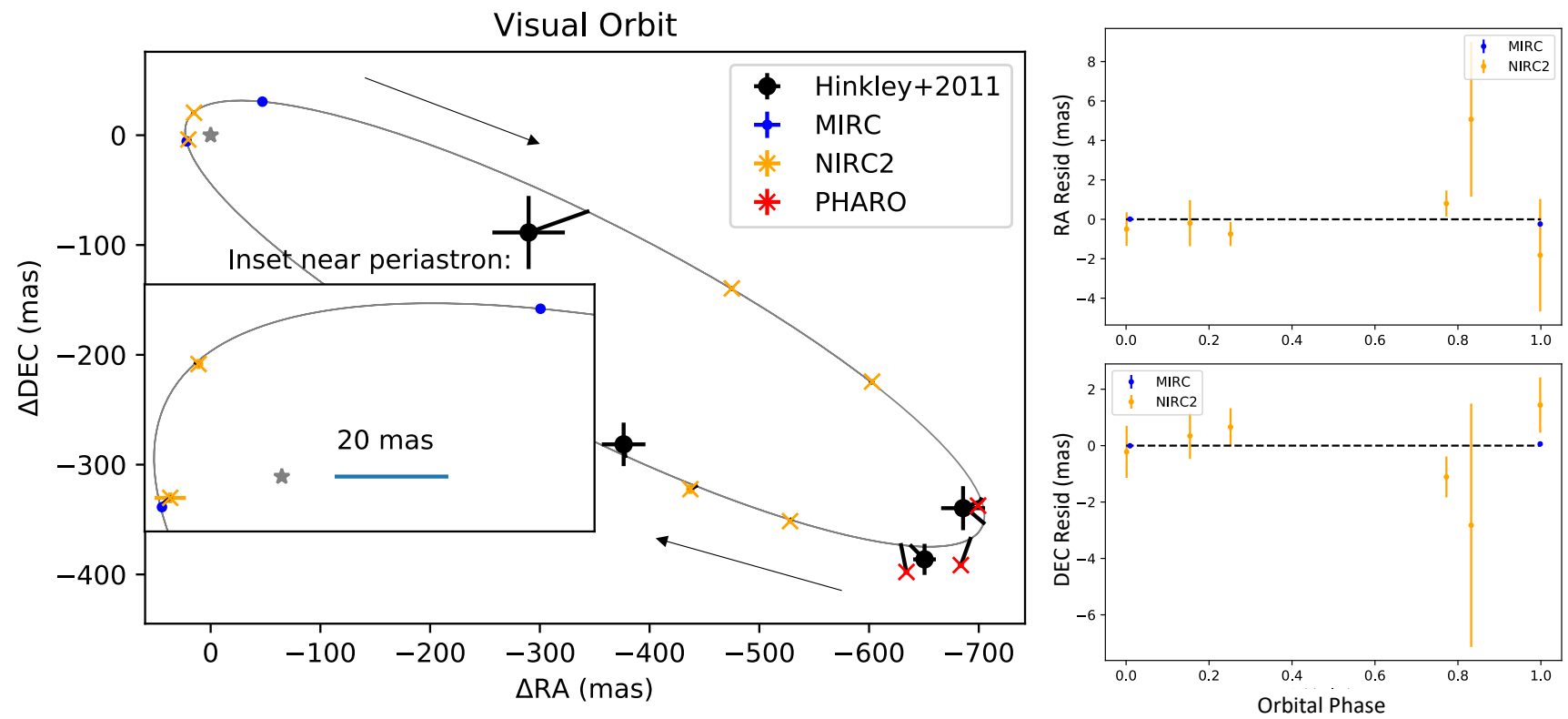

Figure 2. We show the visual orbit for the best fit to the combined visual and spectroscopic data of $\alpha$ Oph. The MIRC and NIRC2 epochs near periastron passage, along with new double-lined RV data, allow for a high precision measure of the masses of this system. We also show the Hinkley et al. (2011) data for comparison, which were not used in our final orbit fit. All of this data fits our orbit well except for the points taken at Palomar Observatory (particularly, those with the PHARO instrument - see the text for details). Including this data in the fit has no significant effect on the best-fit orbital elements, which are constrained with our new high precision data.
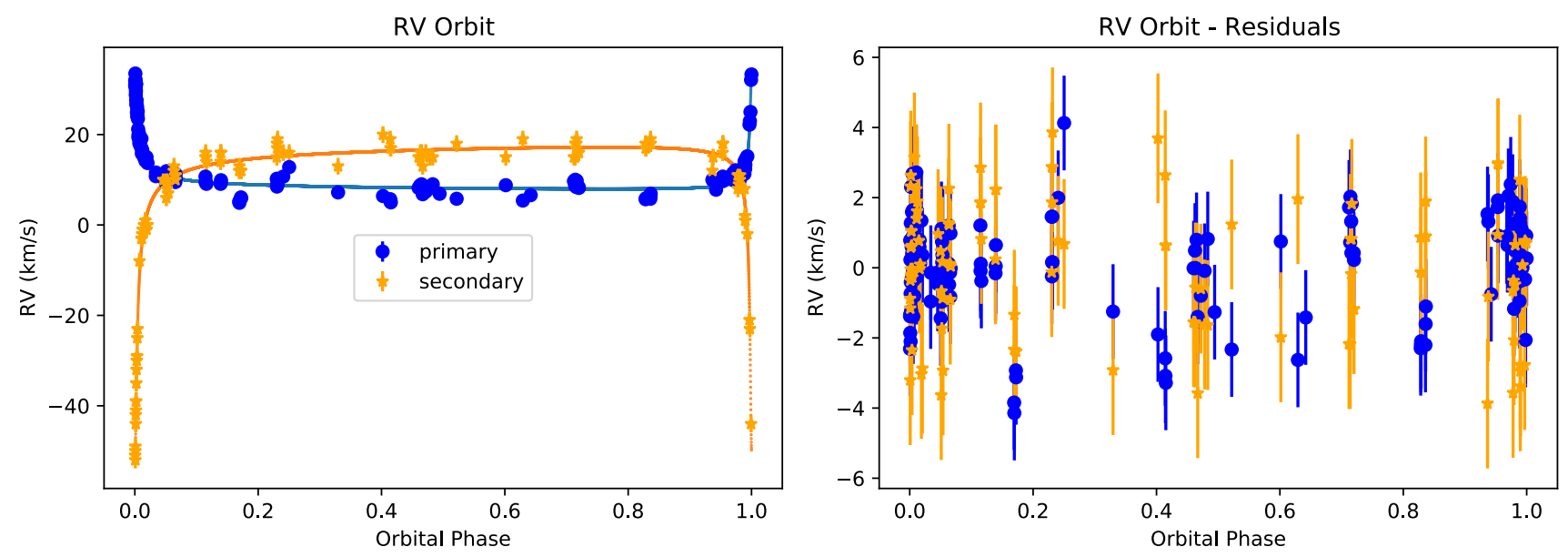

Figure 3. We show the RV orbit of the best fit to the combined visual and spectroscopic data of $\alpha$ Oph. As explained in the text, the different lines used to measure RV lead to different velocity offsets. This does not have a significant effect on the orbit, as one can see from the residuals that the offsets are constant across orbital phase. The shape of the RV curve is also heavily set by the combined fit with the visual orbit. 
Table 3. $\alpha$ Oph: Best fit orbital elements

\begin{tabular}{lcccc}
\hline & Hinkley et al. (2011) & Visual Orbit & RV Orbit & Combined Orbit \\
\hline $\mathrm{P}(\mathrm{d})$ & 3148.4 (fixed) & $3149.6 \pm 5.6$ & $3139.78 \pm 0.35$ & $3139.72 \pm 0.28$ \\
$\mathrm{~T}(\mathrm{JD})$ & $2452888 \pm 53$ & $2456028.20 \pm 0.03$ & $2456028.26 \pm 0.27$ & $2456028.220 \pm 0.029$ \\
$\mathrm{e}$ & $0.92 \pm 0.03$ & $0.93938 \pm 0.00016$ & $0.9370 \pm 0.0012$ & $0.93912 \pm 0.00013$ \\
$\omega(\mathrm{deg})$ & $162 \pm 14$ & $169.98 \pm 0.24$ & $171.18 \pm 0.66$ & $170.21 \pm 0.23$ \\
$\Omega(\mathrm{deg})$ & $232 \pm 9$ & $236.70 \pm 0.17$ & - & $236.86 \pm 0.16$ \\
$\mathrm{i}(\mathrm{deg})$ & $125_{-9}^{+6}$ & $130.67 \pm 0.07$ & - & $130.679 \pm 0.067$ \\
$\mathrm{a}(\mathrm{mas})$ & $427_{-13}^{+20}$ & $410.59 \pm 0.48$ & - & $409.8 \pm 0.3$ \\
$K_{A}(\mathrm{~km} / \mathrm{s})$ & - & - & $12.53 \pm 0.22$ & $12.7 \pm 0.2$ \\
$K_{B}(\mathrm{~km} / \mathrm{s})$ & - & - & $33.35 \pm 0.44$ & $33.74 \pm 0.35$ \\
$\gamma_{A}(\mathrm{~km} / \mathrm{s})$ & - & - & $8.86 \pm 0.14$ & $8.91 \pm 0.14$ \\
$\gamma_{B}(\mathrm{~km} / \mathrm{s})$ & - & - & $14.74 \pm 0.22$ & $14.67 \pm 0.21$ \\
\hline
\end{tabular}

Table 4. $\alpha$ Oph: Physical Properties

\begin{tabular}{lcc}
\hline \hline distance (pc) & $14.80 \pm 0.13$ & This Work \\
$M_{\mathrm{A}}\left(M_{\odot}\right)$ & $2.20 \pm 0.06$ & This Work \\
$M_{\mathrm{B}}\left(M_{\odot}\right)$ & $0.824 \pm 0.023$ & This Work \\
$\Delta$ mag $(\mathrm{Kc})$ & $3.44 \pm 0.12$ & This Work \\
$\Delta$ mag $(\mathrm{Jc})$ & $4.14 \pm 0.08$ & This Work \\
Apparent mag, A (Kband) & $1.684 \pm 0.007$ & This Work+Cohen et al. (1999) \\
Apparent mag, B (Kband) & $5.12 \pm 0.12$ & This Work+Cohen et al. (1999) \\
Apparent mag, A (Jband) & $1.752 \pm 0.005$ & This Work+Cohen et al. (1999) \\
Apparent mag, B (Jband) & $5.89 \pm 0.08$ & This Work+Cohen et al. (1999) \\
$T_{\text {eff,A }}(\mathrm{K})^{\mathrm{a}}$ & $8250 \pm 100$ & Zhao et al. (2009) \\
$L_{A}(\mathrm{~L} \odot)^{\mathrm{a}}$ & $30.2 \pm 1.3$ & Zhao et al. (2009) \\
$R_{\text {pol,A }}$ & $2.390 \pm 0.014$ & Zhao et al. (2009) \\
$R_{\text {eq,A }}$ & $2.871 \pm 0.020$ & Zhao et al. (2009) \\
Age $(\mathrm{Gyr})$ & $0.77 \pm 0.03$ & Zhao et al. (2009) \\
\hline
\end{tabular}

$a_{\text {Averaged over surface }}$

\section{COMPARISON WITH STELLAR EVOLUTION MODELS}

One of the major opportunities offered by high precision binary star orbits is a comparison of observations to stellar evolution models. Zhao et al. (2009) used interferometric imaging and rotator models to measure inclination, equatorial radius and temperature, and rotation speed of $\alpha$ Oph $\mathrm{A}$. Using the true effective temperature and luminosity from those models, we show the position of component A on an HR diagram to compare with its stellar track. We use rotator models from Ekström et al. (2012) to compute isochrones and stellar tracks for the primary, using a rotation rate of $v / v_{\text {crit }}=0.9$ consistent with the high rotation rate in Zhao et al. (2009). We also use Mesa Isochrones and Stellar Tracks (MIST) evolution models to compute tracks without rotation (Dotter 2016; Choi et al. 2016; Paxton et al. 2011, 2013). We assume a solar metallicity in both models.

In Figure 4 it can be seen that $\alpha$ Oph A falls onto a stellar track which agrees with our measured stellar mass within error bars of $2.20 \pm 0.06 \mathrm{M}_{\odot}$ with the rotating-star evolution models. The position on an HR diagram gives an age of about 0.7 Gyr for the system. The fast rotator model is a slightly better fit when compared to a non-rotating evolution model. However, in the non-rotating model a stellar track of $2.11 \mathrm{M}_{\odot}$ still goes through our point, which is $<2-\sigma$ away from our best-fit value given our error bars on mass. To better distinguish between these models, we need a mass measurement at the $\sim 1 \%$ level. The mass of the primary comes from the period, semi-major axis, and distance measurement (which includes the semi-amplitudes and inclination). Currently, distance is the quantity which is limiting our mass precision to just under $3 \%$. We only know distance at the $\sim 1 \%$ level from our RV+visual orbit, 
and the Hipparcos distance has an error bar twice as high as our new value. If the distance were known perfectly (i.e. a fixed quantity), our error on masses would be only $0.6 \%$ given our orbital element precision. Gaia may eventually improve the distance measurement to this system, though this object does not yet have a distance measurement from Gaia. In fact, $\alpha$ Oph is so bright that the current final parallax precision acheived by the end of the Gaia mission is currently unknown. Precision parallax for bright stars was once thought to be unachievable, though many saturation problems have been solved which gives some hope for these brighter targets (Sahlmann et al. 2016). If precision on distance can be improved, we will be able to distinguish between rotating vs non-rotating models on the HR diagram with our precision orbit.
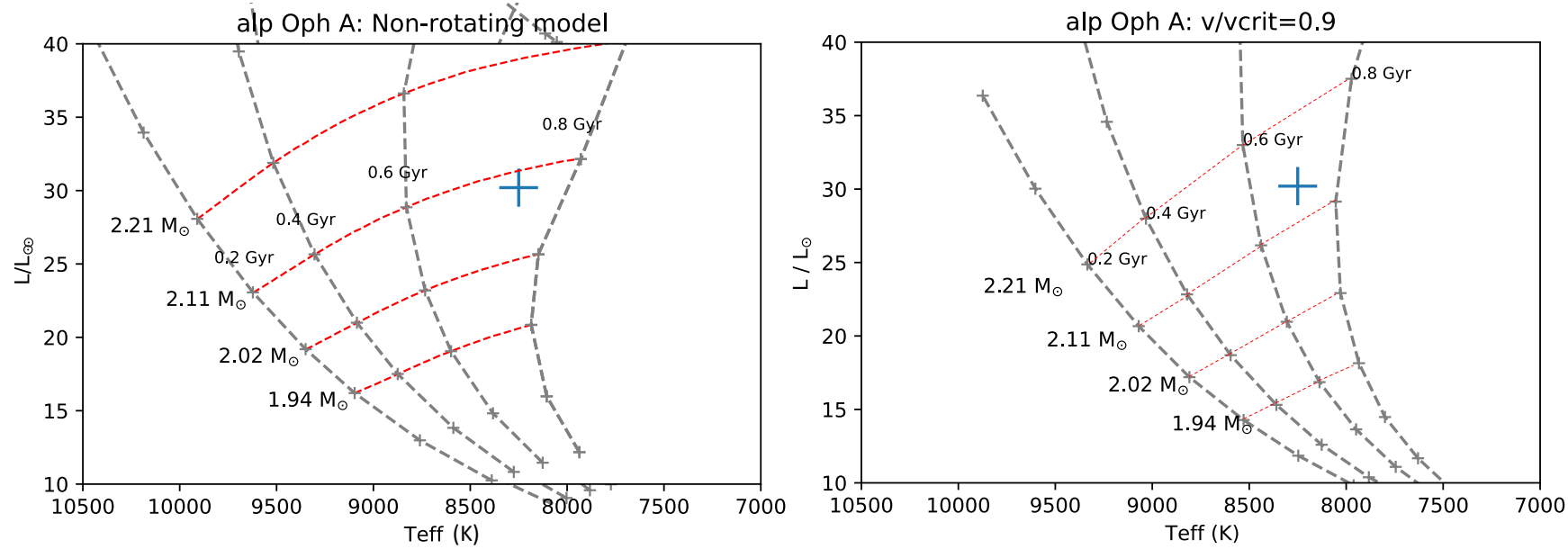

Figure 4. We use stellar models with and without rotation in order to plot $\alpha$ Oph A onto an HR diagram. We show MIST tracks without rotation (left) and Ekström et al. (2012) tracks with $v / v_{\text {crit }}=0.9$ (right). The stellar track with rotation agrees well with our measured mass, and the isochrone implies an age of 0.7 Gyr. The MIST model has more marginal agreement with a $2.11 \mathrm{M}_{\odot}$ track consistent with $\alpha$ Oph's position, a value just outside our 1-sigma error bars.

The secondary component of $\alpha$ Oph does not have a literature value for temperature or luminosity. To measure these values, we use our measured $\mathrm{J}$ and $\mathrm{K}$ flux ratios from Keck AO to compute apparent magnitudes in these bands (presented in Table 4). For apparent magnitudes of the system, Cohen et al. (1999) measure $J=1.728 \pm 0.005$ and $K=1.639 \pm 0.005$. These values also match those measured in Alonso et al. (1994) within error bars. Though we fit a flux ratio in H-band from MIRC data, we have low confidence in its accuracy for precise photometry. This is due to the fact that the companion is near the edge of the interferometric field-of-view for these epochs, where bandwidth smearing can bias the flux ratio measurement. Hence we choose to only report the $\mathrm{J}$ and $\mathrm{K}$ band photometry.

We compute synthetic photometry for the secondary using the MIST models at our measured mass and at solar metallicity. Since we have a measurement of distance for the system and age of the primary, we fix these values. Figure 5 shows the results of this fit. We find that our J-band photometry for component B is slightly under-luminous compared to models with our precisely measured mass for $\alpha$ Oph B. Our fit to the measured apparent magnitudes improves if either 1) the distance is higher, 2) the mass of the secondary is lower, or 3) the metallicity is higher. Due to our high precision orbit, options (1) and (2) seem unlikely. Although we note the potential blending issues of the RV data explained in section 2 which might affect the measured mass ratio. Our mass for the primary is also consistent with that obtained by Hinkley et al. (2011) however, though we note that Gatewood (2005) obtained a slightly lower mass of $0.7778 \pm 0.058 \mathrm{M}_{\odot}$ for the secondary. We show in Figure 5 that a higher metallicity value for $\alpha$ Oph B would make the photometry more consistent with our measured mass. A measurement of metallicity of this component is needed in order to check this consistency. 

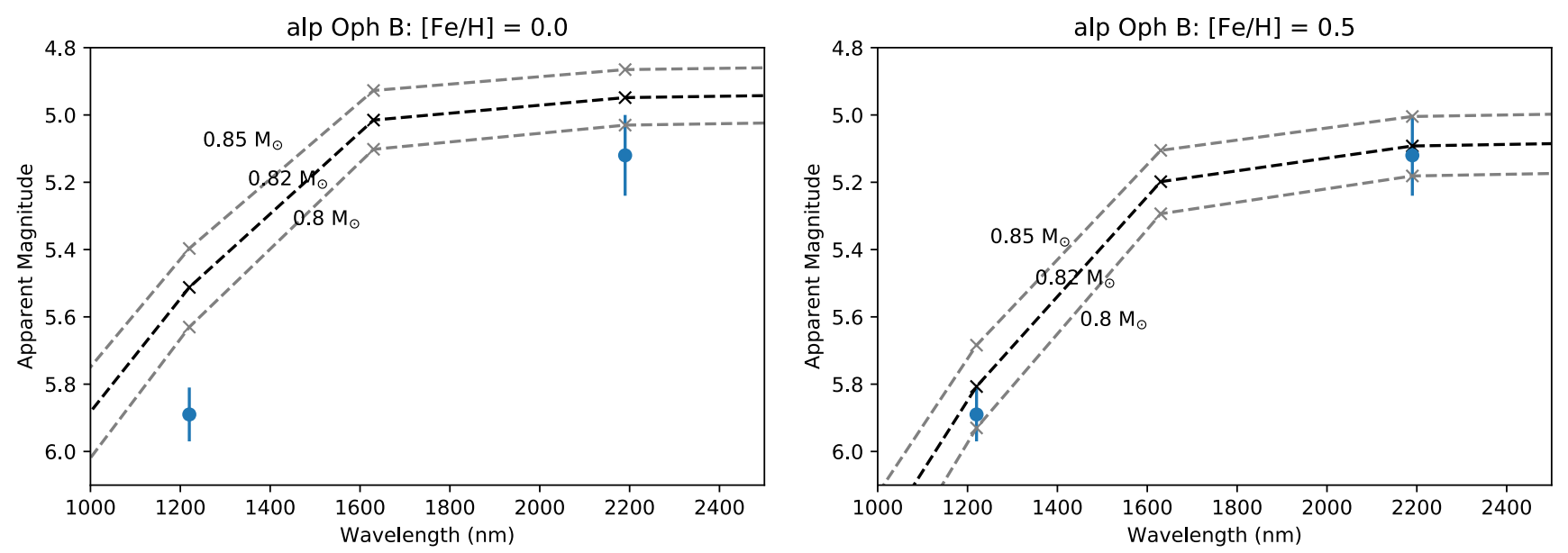

Figure 5. We use apparent $\mathrm{J}$ and $\mathrm{K}$ magnitudes to fit a stellar model for $\alpha$ Oph $\mathrm{B}$. (Left panel) Using MIST models of solar metallicity along with the age and distance measured for the system, we plot the tracks which are consistent with our measured mass and its 1- $\sigma$ uncertainty. This companion is slightly underluminous when compared to stellar models. A larger distance, lower mass of the secondary, or higher metallicity (plotted on right panel) would bring the MIST models into better agreement with our measured mass.

\section{ESTABLISHING $\alpha$ OPH AS AN ASTROMETRIC REFERENCE}

Our new data of the $\alpha$ Oph binary system near periastron passage increases the precision of the system's orbital elements, making this bright source a potential high precision reference source for astrometric programs. High precision binary orbits are useful as calibration sources for instruments to measure wavelength or astrometry calibration. Since wider binaries often have long orbital periods, precision orbits at the level needed by such instruments are often sparse. In Figure 6 we demonstrate our current astrometric precision for this system given our current orbital parameters and their associated uncertainties. We sample orbits from our MCMC posterior distribution and report the 1- $\sigma$ spread about the best-fit orbit at a given time over the next decade. As can be seen in the figure, our orbital element precision was at the $<200 \mu$-as level during the 2012 periastron passage, at $\sim 400 \mu$-as during the 2020 passage, and at a $600-900$ $\mu$-as level for the 2029 periastron passage. Our orbital elements lead to astrometry predictions at a $<1.6$ mas spread across the 8.6-year orbit, with the more precise predictions near periastron passage. This orbital precision can be improved by taking more high precision data outside of periastron passages, which could make this bright binary source an even better astrometric calibration source in the future. 


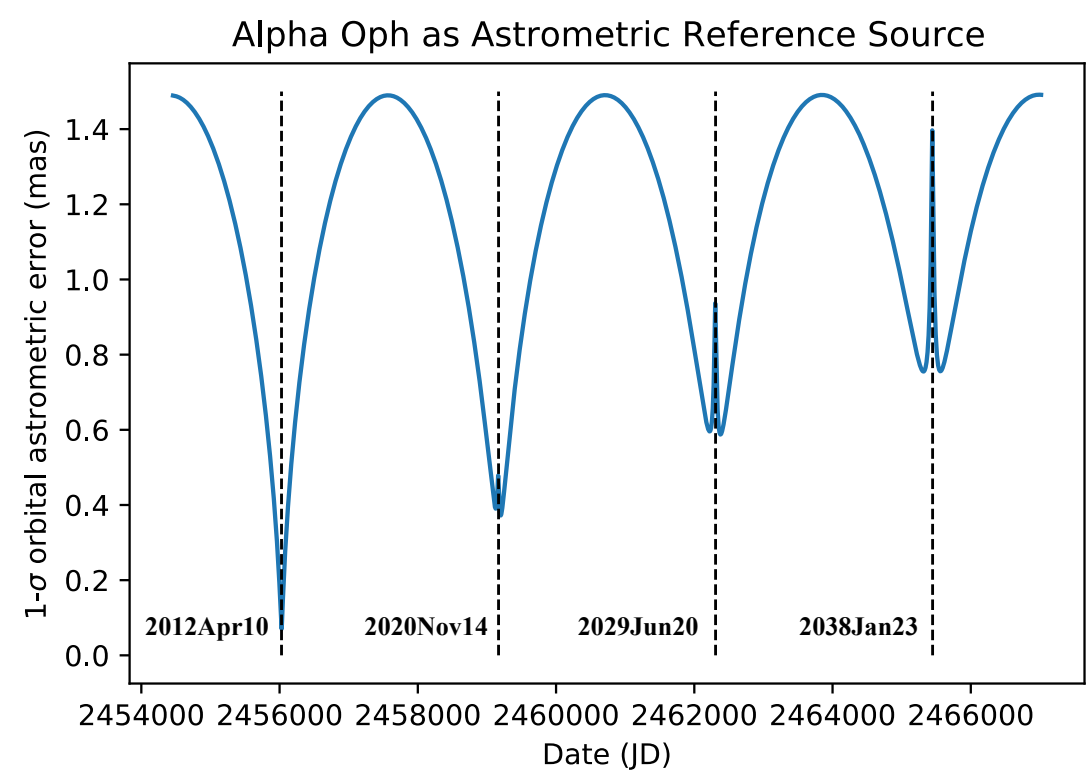

Figure 6. We plot the 2D positional error as a function of observing data for our well-constrained orbit of $\alpha$ Oph. The curve shows our 1- $\sigma$ spread about the best solution at a given date using the posterior chains from the MCMC routine. Astrometric prediction across the next decade are accurate to $<1.5$ mas, with data near periastron approaching the 100 s of $\mu$-as level. Additional high precision data outside of periastron passage will improve this source as a reference over the coming decades.

\section{CONCLUSIONS}

We obtained new interferometric and adaptive optic imaging data on the nearby, bright A5IV binary star $\alpha$ Oph. We also report a new double-lined RV orbit which covers two periastron passages of this eccentric system. Since this binary system is extremely eccentric $(e=0.94)$, data near periastron passage are crucial for improving the orbital elements and mass measurements of this $\sim 8.6$ year period system. Measuring the primary mass at a high precision is especially desired for $\alpha \mathrm{Oph}$, as this star is also a rapid rotator which has been imaged and modeled with high angular resolution interferometric measurements. Visual and RV orbits of binary stars are the only way to directly measure stellar masses to high precision, and we use our well-covered orbit of $\alpha$ Oph to validate mass estimates which come from the modeling of rapidly rotating stars. This system is highly unique in being an imaged rapid rotator and having a well characterized binary orbit. Other imaged rotators such as $\alpha$ Cep and $\beta$ Cas do not have known companions, while the companion for the rapid rotator Regulus is complicated by mass transfer and extremely hard to detect as a visual binary. $\alpha$ Oph provides a benchmark test of rotating evolution models that is not easy to achieve with other known systems. Our updated orbit allows for a strict comparison with rapid rotator models.

Hinkley et al. (2011) previously used adaptive optics imaging data to present an orbit and masses for this system, and that paper called for additional data near periastron passage to better constrain the masses. Our previously unpublished data near periastron passage, as well as a full RV orbit, now allows for high precision comparison of masses with rotator models. We directly measure a primary mass of $2.20 \pm 0.06 \mathrm{M}_{\odot}$, in agreement with the model predictions using rapid rotator imaging results of Zhao et al. (2009). We compare the primary star with stellar evolution models on an HR diagram, and find that it fits well on a track with our measured stellar mass, solar metallicity, and an age of $\sim 0.7$ Gyr. We find that stellar evolution models including rotation give a slightly more consistent fit to our measured mass, though even with our $2.7 \%$ mass error this is a $<2-\sigma$ result. To better distinguish between evolution models, we need $\sim 1 \%$ mass errors. This can be accomplished by improving the error on distance to the system, which Gaia might be able to do in future data releases. The secondary component of the binary system is slightly underluminous for our measured mass of $0.824 \pm 0.023 \mathrm{M}_{\odot}$. This discrepancy might be explained if the metallicity is higher than solar. The mutual inclination of the rotation axis of the star and the orbital plane of the binary is non co-planar at either $42.7 \pm 0.6^{\circ}$ or $133.0 \pm 0.6^{\circ}$, depending on the rotation orientation of the primary. The mutual inclination and high eccentricity of the binary system may hint at early interactions in the star formation stages.

We demonstrate that current orbital precision makes $\alpha$ Oph a potentially useful astrometric calibration source for other instruments. It is bright, easily observable, and the binary can be resolved by single-aperture telescopes. Additional high precision astrometry data away from periastron could improve the orbital elements further, to approach 
$<100 \mu$-as predictive power over the next orbit.

T.G. and J.D.M. acknowledge support from NASA-NNX16AD43G, and from NSF-AST2009489. T.G. acknowledges support from Michigan Space Grant Consortium, NASA grant NNX15AJ20H. S.K. acknowledges support from an ERC Starting Grant (grant agreement no. 639889). Astronomy at Tennessee State University is supported by the state of Tennessee through its Centers of Excellence program. This work is based upon observations obtained with the Georgia State University Center for High Angular Resolution Astronomy Array at Mount Wilson Observatory. The CHARA Array is supported by the National Science Foundation under Grant No. AST-1636624 and AST-1715788. Institutional support has been provided from the GSU College of Arts and Sciences and the GSU Office of the Vice President for Research and Economic Development. Some of the data presented herein were obtained at the W. M. Keck Observatory, which is operated as a scientific partnership among the California Institute of Technology, the University of California and the National Aeronautics and Space Administration. The Observatory was made possible by the generous financial support of the W. M. Keck Foundation. This research has made use of the Keck Observatory Archive (KOA), which is operated by the W. M. Keck Observatory and the NASA Exoplanet Science Institute (NExScI), under contract with the National Aeronautics and Space Administration. The authors wish to recognize and acknowledge the very significant cultural role and reverence that the summit of Maunakea has always had within the indigenous Hawaiian community. We are most fortunate to have the opportunity to conduct observations from this mountain. This research has made use of the Jean-Marie Mariotti Center SearchCal service ${ }^{1}$.

\section{Facilities: CHARA, Fairborn Observatory, Keck:II (NIRC2)}

Software: lmfit, astropy, emcee

\section{REFERENCES}

Alonso, A., Arribas, S., \& Martinez-Roger, C. 1994, A\&AS, 107, 365

Astropy Collaboration, Robitaille, T. P., Tollerud, E. J., et al. 2013, A\&A, 558, A33

Astropy Collaboration, Price-Whelan, A. M., Sipőcz, B. M., et al. 2018, AJ, 156, 123

Augensen, H. J., \& Heintz, W. D. 1992, PASP, 104, 314

Bailey, J., Cotton, D. V., Howarth, I. D., Lewis, F., \& Kedziora-Chudczer, L. 2020, MNRAS, 494, 2254

Boden, A. F., Koresko, C. D., van Belle, G. T., et al. 1999, ApJ, 515,356

Che, X., Monnier, J. D., Kraus, S., et al. 2012, in Society of Photo-Optical Instrumentation Engineers (SPIE) Conference Series, Vol. 8445, Optical and Infrared Interferometry III, ed. F. Delplancke, J. K. Rajagopal, \& F. Malbet, $84450 Z$

Che, X., Monnier, J. D., \& Webster, S. 2010, in Society of Photo-Optical Instrumentation Engineers (SPIE) Conference Series, Vol. 7734, Optical and Infrared Interferometry II, ed. W. C. Danchi, F. Delplancke, \& J. K. Rajagopal, 77342V Chelli, A., Duvert, G., Bourgès, L., et al. 2016, A\&A, 589, A112 Choi, J., Dotter, A., Conroy, C., et al. 2016, ApJ, 823, 102 Cohen, M., Walker, R. G., Carter, B., et al. 1999, AJ, 117, 1864

Domiciano de Souza, A., Kervella, P., Moser Faes, D., et al. 2014, A\&A, 569, A10

Dotter, A. 2016, ApJS, 222, 8

Eaton, J. A., \& Williamson, M. H. 2007, PASP, 119, 886

Ekström, S., Georgy, C., Eggenberger, P., et al. 2012, A\&A, 537, A146

Fekel, F. C., Rajabi, S., Muterspaugh, M. W., \& Williamson, M. H. 2013, AJ, 145, 111

${ }^{1}$ available at http://www.jmmc.fr/searchcal_page.htm
Fekel, F. C., Tomkin, J., \& Williamson, M. H. 2009, AJ, 137, 3900

Fekel, F. C., J. 1981, ApJ, 246, 879

Foreman-Mackey, D., Hogg, D. W., Lang, D., \& Goodman, J. 2013, PASP, 125, 306

Gatewood, G. 2005, AJ, 130, 809

Herbison-Evans, D., Hanbury Brown, R., Davis, J., \& Allen, L. R. 1971, MNRAS, 151, 161

Hinkley, S., Monnier, J. D., Oppenheimer, B. R., et al. 2011, ApJ, 726, 104

Ireland, M. J., Kraus, A., Martinache, F., Lloyd, J. P., \& Tuthill, P. G. 2008, ApJ, 678, 463

Kamper, K. W., Legget, D., \& McCarthy, Donald W., J. 1989, AJ, 98, 686

Kraus, A. L., \& Ireland, M. J. 2012, ApJ, 745, 5

Kraus, A. L., Ireland, M. J., Huber, D., Mann, A. W., \& Dupuy, T. J. 2016, AJ, 152, 8

McCarthy, D. W., J. 1983, in IAU Colloq. 76: Nearby Stars and the Stellar Luminosity Function, ed. A. G. D. Philip \& A. R. Upgren, 107

Monnier, J. D., Townsend, R. H. D., Che, X., et al. 2010, ApJ, 725,1192

Monnier, J. D., Pedretti, E., Thureau, N., et al. 2006, in Proc. SPIE, Vol. 6268, Society of Photo-Optical Instrumentation Engineers (SPIE) Conference Series, 62681P

Monnier, J. D., Zhao, M., Pedretti, E., et al. 2007, Science, 317, 342

Monnier, J. D., Che, X., Zhao, M., et al. 2012, ApJL, 761, L3

Newville, M., Stensitzki, T., Allen, D. B., et al. 2016, Lmfit: Non-Linear Least-Square Minimization and Curve-Fitting for Python, , , ascl:1606.014

Paxton, B., Bildsten, L., Dotter, A., et al. 2011, ApJS, 192, 3

Paxton, B., Cantiello, M., Arras, P., et al. 2013, ApJS, 208, 4

Pope, B., Tuthill, P., Hinkley, S., et al. 2016, MNRAS, 455, 1647 
Rizzuto, A. C., Dupuy, T. J., Ireland, M. J., \& Kraus, A. L. 2020, ApJ, 889, 175

Royer, F., Grenier, S., Baylac, M. O., Gómez, A. E., \& Zorec, J. 2002, A\&A, 393, 897

Sahlmann, J., Martín-Fleitas, J., Mora, A., et al. 2016, in Proc. SPIE, Vol. 9904, Space Telescopes and Instrumentation 2016: Optical, Infrared, and Millimeter Wave, 99042E

ten Brummelaar, T. A., McAlister, H. A., Ridgway, S. T., et al. 2005, The Astrophysical Journal, 628, 453 van Belle, G. T. 2012, A\&A Rv, 20, 51

van Leeuwen, F. 2007, A\&A, 474, 653

Willson, M., Kraus, S., Kluska, J., et al. 2016, A\&A, 595, A9

Wright, J. T., \& Howard, A. W. 2009, ApJS, 182, 205

Yelda, S., Lu, J. R., Ghez, A. M., et al. 2010, ApJ, 725, 331

Zhao, M., Monnier, J. D., Pedretti, E., et al. 2009, ApJ, 701, 209 


\section{APPENDIX}

\section{A. RADIAL VELOCITY DATA}

Table A1. $\alpha$ Oph Radial Velocities

\begin{tabular}{|c|c|c|}
\hline $\mathrm{HJD}^{\mathrm{a}}$ & $R V_{A}\left(\mathrm{~km} \mathrm{~s}^{-1}\right)^{\mathrm{b}}$ & $R V_{B}\left(\mathrm{~km} \mathrm{~s}^{-1}\right)^{\mathrm{c}}$ \\
\hline 2455847.6533 & 7.8 & - \\
\hline 2455927.0572 & 10.2 & - \\
\hline 2455930.0570 & 10.0 & - \\
\hline 2455935.0558 & 11.5 & - \\
\hline 2455947.0524 & 12.1 & - \\
\hline 2455957.0473 & 11.9 & - \\
\hline 2455967.0446 & 10.9 & - \\
\hline 2455976.0409 & 10.8 & - \\
\hline 2455991.0334 & 12.4 & - \\
\hline 2456001.0269 & 14.8 & - \\
\hline 2456017.9313 & 22.2 & -21 \\
\hline 2456019.9748 & 23.0 & -23 \\
\hline 2456022.8985 & 25.0 & - \\
\hline 2456025.9039 & 32.1 & -44 \\
\hline 2456028.0124 & 33.3 & - \\
\hline 2456029.8003 & 30.9 & -51 \\
\hline 2456029.9008 & 31.8 & - \\
\hline 2456030.8395 & 31.4 & -50 \\
\hline 2456030.9554 & 30.8 & -49 \\
\hline 2456032.9811 & 28.8 & -44 \\
\hline 2456033.8286 & 31.3 & -39 \\
\hline 2456035.0076 & 31.1 & - \\
\hline 2456037.0024 & 27.6 & -32 \\
\hline 2456038.0022 & 26.5 & -30 \\
\hline 2456045.0015 & 21.2 & - \\
\hline 2456046.0008 & 19.4 & - \\
\hline 2456046.9992 & 20.0 & - \\
\hline 2456048.9992 & 20.3 & - \\
\hline 2456050.9982 & 18.1 & - \\
\hline 2456051.9965 & 18.8 & -8 \\
\hline 2456052.9940 & 19.4 & - \\
\hline 2456055.9947 & 17.6 & - \\
\hline 2456060.9922 & 17.1 & - \\
\hline 2456061.8736 & 19.1 & -3 \\
\hline 2456063.8189 & 15.8 & -3 \\
\hline 2456065.8098 & 16.6 & -2 \\
\hline 2456067.7947 & 16.0 & -1 \\
\hline 2456074.7881 & 15.0 & 0 \\
\hline 2456077.9448 & 15.0 & 0 \\
\hline 2456078.9837 & 15.1 & - \\
\hline 2456081.9150 & 14.2 & 1 \\
\hline 2456087.7671 & 15.0 & -1 \\
\hline
\end{tabular}


Table A1. $\alpha$ Oph Radial Velocities

\begin{tabular}{|c|c|c|}
\hline HJD $^{\mathrm{a}}$ & $R V_{A}\left(\mathrm{~km} \mathrm{~s}^{-1}\right)^{\mathrm{b}}$ & $R V_{B}\left(\mathrm{~km} \mathrm{~s}^{-1}\right)^{\mathrm{c}}$ \\
\hline 2456092.8878 & 13.7 & 0 \\
\hline 2456134.8539 & 10.8 & - \\
\hline 2456135.6749 & 11.6 & - \\
\hline 2456170.7241 & 10.6 & - \\
\hline 2456172.7161 & 10.9 & 10 \\
\hline 2456185.6536 & 9.4 & 10 \\
\hline 2456188.6476 & 10.7 & 6 \\
\hline 2456189.7018 & 11.9 & 9 \\
\hline 2456191.6455 & 9.8 & 8 \\
\hline 2456192.6322 & 11.5 & - \\
\hline 2456193.6779 & 10.9 & - \\
\hline 2456194.6758 & 11.1 & 10 \\
\hline 2456195.6820 & 10.4 & 9 \\
\hline 2456196.6412 & 11.6 & - \\
\hline 2456197.7243 & 10.4 & 7 \\
\hline 2456226.5981 & 11.6 & 12 \\
\hline 2456227.5979 & 10.5 & 13 \\
\hline 2456228.5831 & 9.9 & 12 \\
\hline 2456233.5810 & 10.2 & 11 \\
\hline 2456234.5802 & 9.5 & 10 \\
\hline 2456235.5796 & 11.3 & - \\
\hline 2456236.5789 & 10.3 & 11 \\
\hline 2456387.9042 & 10.7 & 15 \\
\hline 2456388.7848 & 9.4 & 16 \\
\hline 2456389.7884 & 9.6 & 15 \\
\hline 2456392.7780 & 9.1 & 14 \\
\hline 2456464.7862 & 9.1 & 14 \\
\hline 2456465.7862 & 9.3 & 16 \\
\hline 2456466.7862 & 9.9 & 16 \\
\hline 2456559.6290 & 5.2 & 12 \\
\hline 2456560.6282 & 4.9 & 13 \\
\hline 2456568.6220 & 6.1 & 12 \\
\hline 2456569.6218 & 5.9 & 12 \\
\hline 2456750.8676 & 10.2 & 15 \\
\hline 2456751.7894 & 8.5 & 18 \\
\hline 2456752.8030 & 8.9 & 17 \\
\hline 2456753.8034 & 10.2 & 18 \\
\hline 2456754.7838 & 8.9 & 19 \\
\hline 2456783.8659 & 10.7 & 16 \\
\hline 2456813.9677 & 12.8 & 16 \\
\hline 2457062.9706 & 7.2 & 13 \\
\hline 2457291.6473 & 6.4 & 20 \\
\hline 2457328.5954 & 5.7 & 19 \\
\hline 2457329.6022 & 5.2 & 17 \\
\hline 2457331.5911 & 5.0 & 17 \\
\hline
\end{tabular}


Table A1. $\alpha$ Oph Radial Velocities

\begin{tabular}{|c|c|c|}
\hline HJD $^{\mathrm{a}}$ & $R V_{A}\left(\mathrm{~km} \mathrm{~s}^{-1}\right)^{\mathrm{b}}$ & $R V_{B}\left(\mathrm{~km} \mathrm{~s}^{-1}\right)^{\mathrm{c}}$ \\
\hline 2457472.9618 & 8.2 & 15 \\
\hline 2457479.8958 & 8.7 & 15 \\
\hline 2457481.9314 & 8.2 & 16 \\
\hline 2457487.8848 & 9.0 & 16 \\
\hline 2457493.8473 & 6.8 & 13 \\
\hline 2457509.7589 & 7.4 & 16 \\
\hline 2457528.9745 & 8.1 & 15 \\
\hline 2457544.9048 & 9.0 & 15 \\
\hline 2457579.9297 & 6.9 & - \\
\hline 2457666.6528 & 5.8 & 18 \\
\hline 2457916.8663 & 8.8 & 15 \\
\hline 2458003.7437 & 5.4 & 19 \\
\hline 2458043.6544 & 6.6 & - \\
\hline 2458263.8664 & 9.7 & 15 \\
\hline 2458269.6760 & 8.7 & 15 \\
\hline 2458270.8365 & 10.0 & 18 \\
\hline 2458274.8666 & 9.3 & 17 \\
\hline 2458275.8466 & 8.4 & 18 \\
\hline 2458277.8466 & 9.8 & 19 \\
\hline 2458287.8366 & 8.4 & 16 \\
\hline 2458288.8366 & 8.2 & 16 \\
\hline 2458628.6836 & 5.7 & 18 \\
\hline 2458629.6841 & 5.8 & 17 \\
\hline 2458630.6872 & 5.9 & 17 \\
\hline 2458652.7964 & 5.8 & 18 \\
\hline 2458653.7964 & 6.4 & 19 \\
\hline 2458654.7964 & 6.9 & 18 \\
\hline 2458968.7391 & 10.0 & 12 \\
\hline 2458972.9149 & 9.8 & 15 \\
\hline 2459020.6609 & 10.5 & 16 \\
\hline 2459021.6614 & 10.7 & 18 \\
\hline 2459022.6610 & 9.7 & 18 \\
\hline 2459097.6584 & 11.3 & 11 \\
\hline 2459098.6461 & 9.9 & 8 \\
\hline 2459100.6702 & 9.9 & 11 \\
\hline 2459103.6290 & 9.1 & 9 \\
\hline 2459108.6256 & 10.0 & 10 \\
\hline 2459109.6239 & 10.6 & 11 \\
\hline 2459110.6197 & 10.5 & - \\
\hline 2459131.6945 & 11.3 & - \\
\hline 2459132.5989 & 14.1 & 8 \\
\hline 2459133.5976 & 13.9 & - \\
\hline 2459134.5970 & 13.9 & 2 \\
\hline 2459135.5958 & 12.4 & 1 \\
\hline 2459136.6397 & 13.3 & 1 \\
\hline
\end{tabular}


Table A1. $\alpha$ Oph Radial Velocities

\begin{tabular}{llc}
\hline $\mathrm{HJD}^{\mathrm{a}}$ & $R V_{A}\left(\mathrm{~km} \mathrm{~s}^{-1}\right)^{\mathrm{b}}$ & $R V_{B}\left(\mathrm{~km} \mathrm{~s}^{-1}\right)^{\mathrm{c}}$ \\
\hline 2459146.6325 & 15.2 & -2 \\
2459170.5849 & 33.5 & -52 \\
2459171.5639 & 32.2 & - \\
2459172.5635 & 30.3 & -42 \\
2459173.5632 & 29.6 & -41 \\
2459174.5625 & 27.7 & -39 \\
2459175.5622 & 26.7 & -35 \\
2459178.5577 & 24.9 & -29 \\
2459179.5566 & 26.0 & -29 \\
2459180.5549 & 24.1 & -25 \\
2459181.5536 & 23.9 & -23 \\
2459182.5519 & 25.1 & - \\
2459183.5501 & 23.5 & - \\
\hline
\end{tabular}

${ }^{a}$ HJD $=$ Heliocentric Julian Date

${ }^{b}$ Errors on primary are $1.4 \mathrm{~km} \mathrm{~s}^{-1}$.

${ }^{c}$ Errors on secondary are $2 \mathrm{~km} \mathrm{~s}^{-1}$.

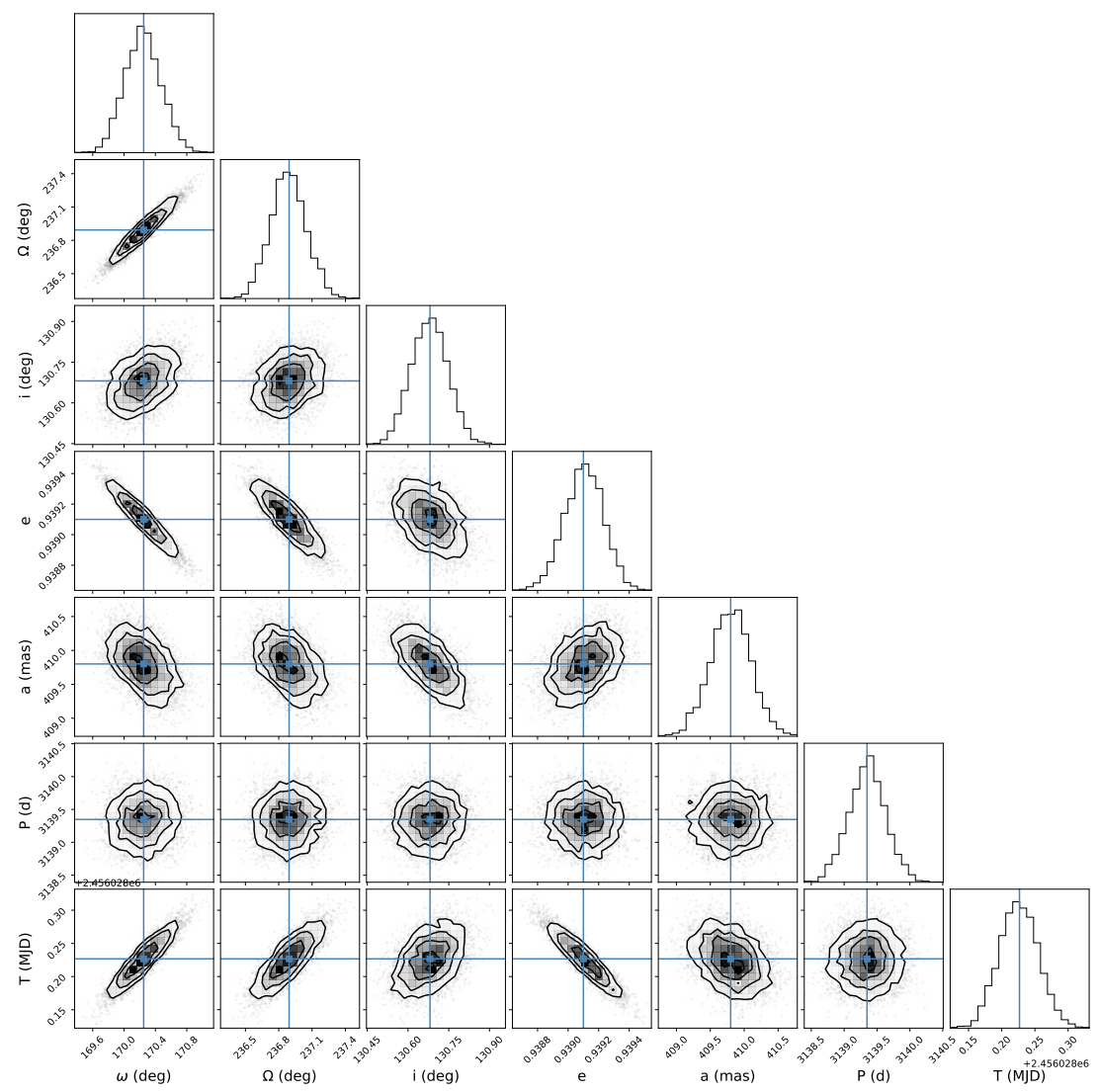

Figure A1. We show the corner plot of posterior distributions from our MCMC routine to estimate errors on the orbital parameters. Since the RV parameters do not show significant correlations, we include only the visual orbital elements for clarity. There are correlations of the time of periastron passage with the elements of eccentricity, $\omega$, and $\Omega$ which is normal for eccentric orbits. 